ARTÍCULO ORIGINAL

\title{
Perfil profesional del contador público colegiado y su influencia en la detección de los actos de corrupción en la región Moquegua
}

\author{
Professional profile of the Certified Public Accountant and his influence \\ on the detection of the acts of corruption in the Moquegua region
}

\begin{abstract}
RESUMEN
El objetivo del estudio realizado en el año 2019, fue determinar si el perfil profesional del contador público colegiado influye en la detección de los actos de corrupción en la región Moquegua. Al considerar que, hoy en día, la región Moquegua es una de las regiones con más casos de corrupción a nivel nacional. Al respecto, el Estado y las empresas privadas, han adoptado medidas, sin embargo, no consideran al profesional en Contabilidad para contrarrestar la corrupción, teniendo conocimiento que el Contador se desenvuelve en los escenarios donde se genera en su mayoría los actos de corrupción. A fin de lograr el objetivo planteado, el estudio se situó como una investigación básica de nivel descriptivo y explicativo, no experimental y transversal, utilizando el modelo de regresión logística Logit, donde la variable dependiente es dicotómica y las variables independientes se encuentran en una escala de intervalo. Como resultado, se determinó que el perfil profesional del contador público colegiado, que posee experiencia profesional de seis a diez años, con especialización de Contador General, especialista en Finanzas, Tributaria, Normas Internacionales de Información Financiera, Costos, Presupuestos, Integrador Contable y Auditor, con capacitación constante de 501 a más horas en los últimos cinco años y que se encuentra en el rango de edad de 31 a 45 años, influye significativamente en la detección de actos de corrupción en la región Moquegua.
\end{abstract}

Bethy Verlina Arohuanca Antahuanaco

Colegio de Contadores Públicos de Moquegua

Moquegua, Perú

bethyaroa@gmail.com

https://orcid.org/0000-0003-4447-0242

\section{RESUMEN}

Palabras clave: Perfil, profesional, contador, detección, actos, corrupción.

\begin{abstract}
The objective of the study was to determine whether the professional profile of the certified public accountant influences the detection of acts of corruption in the Moquegua region. Considering that, nowadays, the Moquegua region is one of the regions with more cases of corruption nationwide. In this regard, the State and private companies have adopted measures, they do not consider the professional accountant to counteract corruption. In order to achieve the proposed objective, the study was placed as a basic research of descriptive and explanatory level, not experimental and transversal, using the logistic regression model Logit was used, where the dependent variable is dichotomous and the independent variables are in an interval scale. As a result, it was determined that the professional profile of the certified public accountant, that has professional experience of six to ten years, specializing in General Accountant, specialist in Finance, Tax, International Financial Reporting Standards, Costs, Budgets, Accounting Integrator and Auditor, with constant training from 501 to more hours in the last five years and who is in the age range of 31 to 45 years, significantly influences the detection of acts of corruption in the Moquegua region.
\end{abstract}

Keywords: Profile professional, accountant, detection, acts, corruption. 


\section{INTRODUCCIÓN}

Frente a la coyuntura mundial donde diariamente la corrupción es una forma de hacer negocios exitosos en las diversas economías y sistemas políticos, con la participación de los trabajadores y terceros vinculados a la entidad pública, privada y mixta, en forma independiente o sistematizada, en perjuicio de las grandes mayorías, perjudicando social y económicamente el desarrollo de una ciudad, de un país y el mundo en general. El presente estudio pretende plantear alternativas de solución, toda vez que la región Moquegua también se ha visto muy afectada por la corrupción constituyéndose actualmente como una de las regiones con más casos de corrupción a nivel nacional. En tal sentido, la investigación realizada presenta el perfil profesional del contador público colegiado que influye significativamente en la detección de los actos de corrupción, el mismo que se encuentra fundamentado con el marco teórico correspondiente.

En relación a la corrupción, se destaca lo señalado por Guillen (2017) que describe la corrupción como un fenómeno que impone ineficacia, pobreza e injusticia a las diversas economías y sistemas políticos, ya que sus ramificaciones se extienden por todo el mundo, no se trata de un rasgo propio de algunos gobiernos subdesarrollados y no solo corresponde a esa área geográfica como podría pensarse. Afirmación que es complementada por Luis (2012), quien refiere que el fenómeno de la corrupción es el peor de todos los males, que sin lugar a dudas aquejan a la sociedad, con efectos perversos y nocivos que se encuentran incrustados cada vez más en la vida nacional. Asimismo, Basílico (2014) especifica en detalle sobre el sistema de corrupción institucionalizado, señalando que la corrupción desarrolla un sistema organizativo secreto, virtual y paralelo, ya existentes en las organizaciones de las cuales se nutre. Supone la existencia de actores (lícitos e ilícitos) que operan en diferentes funciones y niveles, y que constituye entre sí, una red de complicidades (o pacto de silencio) y contratos tácitos (o procesos transaccionales) que involucran algún tipo de contraprestación infractora.

Finalmente, para Fernández (2012), los hechos ilícitos se encuentran conformados por la participación de un (elemento subjetivo necesario) agente público, de la obtención de un beneficio ilícito (elemento causal) y del incumplimiento de un deber de servicio (elemento prescriptivo); y elemento material de la ejecución de un daño a la Administración pública con carácter contingente, de una nota de clandestinidad (elemento formal) y de la participación de un particular (elemento subjetivo contingente). Hechos que podrían ser contrarrestados por el contador público colegiado, tal como lo manifiesta Alegría (2016), quien indica que el contador público tiene como obligación velar por los intereses económicos de la colectividad, dado que es un factor de directa y activa intervención en las organizaciones privadas y públicos, no solamente a las personas jurídicas o naturales relacionadas directamente a la institución sino a la ciudadanía en general y el Estado.

De igual forma, Santos (2018) refiere que el contador público desempeña un rol preponderante: es el profesional presente en todas las instituciones privadas o públicas, responsable de las cuentas y sobre quién recae el pasivo de la sensación pública de las inseguridades, asimismo, destaca que su motivo de ser son los balances justos y es el depositario de la confianza racional, puesto que es quien garantiza el manejo limpio del dinero y la inversión. Estas concepciones se pueden convertir en una herramienta esencial para la lucha contra la corrupción, si suma lo referido por Hernández (2011) en relación al perfil profesional que, es el conjunto de capacidades y competencias de una persona para asumir con responsabilidad el desarrollo de tareas y funciones de una determinada profesión, en ese entender, al profesional en Contabilidad se debe adicionar experiencia profesional, capacitación, especialización, género y rango de edad adecuado.

La experiencia profesional, según Cart \& Toutin (1998), permite adquirir competencias de la elasticidad y la variabilidad de los empleos, en el proceso de construcción de las competencias juega un papel esencial la diversificación de la actividad profesional. De igual forma, la especialización de conocimientos, según Zapata \& Sigala (2010), está relacionada con la variedad, cantidad y profundidad 
de habilidades y conocimientos que un individuo debe poseer para responder a empleos y/o trabajos con un alto grado de dificultad e incertidumbre para ejecutarlos y entenderlos, o simplemente para responder apropiadamente con capacidad suficiente a la variedad de dificultades que se le presente en su trabajo. En lo referente a la capacitación, Maturana (1997) señala que, es la adquisición de capacidades y habilidades de acción fundamental para la ampliación de la inteligencia y la posibilidad de darle un sentido adecuado a lo que se aprende y al aprender, se crea un ámbito de intensificación de las capacidades de hacer, que consiste en la creación de espacios de labor donde se practiquen las habilidades que se ansía desarrollar con la creación efectiva de los espacios de acción involucrados. En relación a la edad, de acuerdo a lo manifestado por Valpuesta (2003), se desarrolla conforme se desenvuelve en la etapa normal de su formación, asimismo, marca las fases en el desarrollo y evolución de la persona. Desde esta configuración, está claro que para la argumentación jurídica no puede ser lo mismo una persona madura de 40 años y un niño de 3 años. En lo referente al género, según Aldana (2013), entre mujeres y hombres no hay diferencia significativa en cuanto al grado de compromiso organizacional. Finalmente, Lamas (2000) señala que el género es un concepto taxonómico útil para clasificar a qué tipo, especie o clase pertenece algo o alguien, como cuando se habla de hombres y mujeres como género femenino y masculino que constituye personas con un sexo común.

Una vez conceptualizada la corrupción y analizada la función que cumple el contador público colegiado en las entidades públicas, privadas, mixtas y en la sociedad en general, así como, la experiencia profesional, especialización, capacitación, edad y género del profesional en Contabilidad, se procede a relacionar dichas variables, toda vez que el contador, según sus competencias y capacidades, se desempeña profesionalmente en las diferentes áreas y niveles del gobierno central, gobiernos regionales, municipalidades, empresas del estado, unidades ejecutoras, bancos, empresas comerciales y de servicios, minerías, agrícolas, industriales, consultoras, contratistas, entre otras instituciones y/o empresas que requieran de sus servicios, dónde precisamente se produce la transgresión activa o pasiva del deber encargado, inobservancia de las normativas pertinentes, favorecimientos, sobornos, fraudes, negociaciones incompatibles entre otros delitos, razón por la que, el profesional en Contabilidad juega un papel fundamental en la detección de los actos de corrupción. Por lo tanto, el estudio plantea como hipótesis que el perfil profesional del contador público colegiado influye significativamente en la detección de los actos de corrupción en la región Moquegua y de igual forma plantea como hipótesis específicas que, la experiencia profesional, especialización, capacitación, edad y género del contador público colegiado influyen significativamente en la detección de los actos de corrupción en la región Moquegua, 2019.

\section{MATERIAL Y MÉTODOS}

La investigación se considera básica y recibe el nombre de investigación pura y científica fundamental, debido a que profundiza las variables de estudio. Del mismo modo, el nivel de investigación es el nivel explicativo y descriptivo, porque busca especificar las propiedades y características, los perfiles de grupos, personas, procesos, objetos o cualquier otro fenómeno dirigido a responder por las causas de los fenómenos y eventos físicos o sociales que se someta a un análisis, enfocándose en explicar en qué condiciones se manifiesta y por qué ocurre un fenómeno, o por qué se relacionan dos o más variables. El diseño de investigación es no experimental, toda vez, que no se puede realizar cambios deliberadamente de las variables independientes y es transversal porque recolectan los datos en un solo momento, con el propósito de analizar su incidencia, interrelación y describir variables (Hernández, Fernández \& Baptista 2014).

Para efectos de comprobar las hipótesis planteadas en la investigación se utilizó el modelo de regresión logística Logit, donde la variable dependiente es dicotómica y las variables independientes se encuentran en una escala de intervalo, modelo utilizado para determinar probabilidades apropiadas en las ciencias sociales. 
La población para el presente estudio son los 667 contadores públicos colegiados del Colegio de Contadores Públicos de la región Moquegua, registrados como miembros habilitados hasta el año 2019, quienes se encuentran activos en el campo profesional de la región de forma independiente o dependiente de alguna institución pública, privada o empresas del estado, conforme se detalla en el siguiente cuadro:

Tabla 1

Miembros activos del Colegio de Contadores de Moquegua

\begin{tabular}{cl}
\hline Cantidad de miembros & \multicolumn{1}{c}{ Situación actual } \\
\hline 773 & Miembros inscritos en el padrón de colegiados \\
6 & (-) Finados \\
6 & (-) Retirados \\
2 & (-) Suspensión temporal \\
92 & (-) En proceso de depuración \\
667 & Total miembros colegiados activos \\
\hline
\end{tabular}

Nota. Datos obtenidos del Colegio de Contadores Públicos de la región Moquegua (2020)

La muestra del estudio se calculó con la siguiente fórmula:

$$
n=\frac{N Z^{2} p q}{(N-1) E^{2}+Z^{2} p q}
$$

Dónde:

$\mathrm{N}=$ Población (667)

$\mathrm{Z}=$ Nivel de confianza deseado (1.95)

$\mathrm{p}=$ Proporción de la población con la característica deseada (éxito) $(95 \%)$

$\mathrm{q}=$ Proporción de la población sin la característica deseada (fracaso) $(5 \%)$

$\mathrm{E}=$ Margen de error $(5 \%)$

$\mathrm{n}=$ Muestra calculada (242)

Efectuado el cálculo correspondiente, la muestra calculada para la investigación es de 242 contadores públicos colegiados de la región Moquegua, se consideró un nivel de confianza de $1.95 \%$ y un margen de error de $5 \%$, asimismo, es necesario mencionar que la muestra determinada es de carácter probabilístico, con el objetivo que sea representativa de la población, donde existe la probabilidad de que todos los elementos sean elegidos, este tipo de muestreos en estudios cualitativos son comúnmente por muestreo aleatorio simple, donde se elige al encuestado al azar.

\section{RESULTADOS}

Al considerar que la investigación plantea el perfil profesional del contador público colegiado para la detección de los hechos ilícitos (corrupción) en la región Moquegua, se estableció como fuente primaria de información al contador público colegiado de la región Moquegua. A fin de determinar el perfil profesional del contador que influye significativamente en la detección de la corrupción, se analiza sus años de experiencia profesional, ramas de especialización, horas de capacitación, rango de edad y género del profesional en Contabilidad. En tal sentido, de la población total de contadores públicos colegiados de la región Moquegua, se procesó la información recolectada en forma aleatoria simple de 242 contadores como muestra de la población total, que fueron categorizados para sintetizar el análisis de los datos y mejor entendimiento por cada perfil. El resultado de participación por cada perfil y categoría se detalla a continuación: 


\section{Tabla 2}

Perfil profesional del contador en la región Moquegua, en el año 2019 (participación por perfil y categoría)

\begin{tabular}{|c|c|c|}
\hline \multirow[b]{2}{*}{ Pertil protesional del contador colegiado } & \multicolumn{2}{|c|}{ Total } \\
\hline & $\mathrm{n}^{\circ}$ & $\%$ \\
\hline \multicolumn{3}{|l|}{ Años de experiencia } \\
\hline No tengo experiencia profesional $(0 \mathrm{a}>)$ & 5 & 2.07 \\
\hline$[1$ a $5>$ & 133 & 54.96 \\
\hline$[6$ a $10>$ & 65 & 26.86 \\
\hline De 11 a más años $(<$ a 11$)$ & 39 & 16.12 \\
\hline \multicolumn{3}{|l|}{ Especialización del contador público colegiado } \\
\hline $\begin{array}{l}\text { Gestión administrativa (tesorero, liquidador financiero, } \\
\text { logístico, proyectos de inversión) }\end{array}$ & 161 & 66.53 \\
\hline Auditor & 21 & 8.68 \\
\hline $\begin{array}{l}\text { Contador General (Finanzas, Tributaria, NIIF, Costos y } \\
\text { Presupuestos, Integrador Contable) }\end{array}$ & 57 & 23.55 \\
\hline Perito & 3 & 1.24 \\
\hline \multicolumn{3}{|l|}{ Horas de capacitación (últimos 5 años) } \\
\hline No cuento con capacitación ( 0 a > horas $)$ & 13 & 5.37 \\
\hline Poco $[1$ a $100>$ & 75 & 30.99 \\
\hline Regular [101 a 500> & 108 & 44.63 \\
\hline Constante < a 501 horas & 46 & 19.01 \\
\hline \multicolumn{3}{|l|}{ Edad del contador público colegiado } \\
\hline De 61 a más años $(<$ a 61$)$ & 3 & 1.24 \\
\hline$[23$ a $30>$ & 70 & 28.93 \\
\hline$[31$ a $45>$ & 140 & 57.85 \\
\hline$[46$ a $60>$ & 29 & 11.98 \\
\hline \multicolumn{3}{|l|}{ Género del contador público colegiado } \\
\hline Masculino & 89 & 36.78 \\
\hline Femenino & 153 & 63.22 \\
\hline
\end{tabular}

Nota. Datos extraídos del cuestionario dirigido al contador público colegiado de la región Moquegua (2020)

Esquematizada la información recolectada del profesional colegiado en Contabilidad de la región Moquegua por perfil profesional y categoría, se redefine que la variable independiente se presenta en escala de intervalo y son cinco variables específicas que han sido objeto de estudio en relación a la interrogante planteada ¿Usted en el ejercicio de la profesión contable detecta: favorecimientos en las adquisiciones, direccionamientos, precios sobrevalorados, evasiones fiscales, malversación de fondos, fraudes, patrocinios y otros actos ilícitos en perjuicio de la entidad y el Estado?, que presenta solamente dos alternativas de respuesta: sí detecta y no detecta (interrogante cerrada), relacionada a la detección de hechos de corrupción en la región Moquegua, que constituye la variable dependiente, variable que, por la característica de las alternativas de la respuesta planteada se configura como dicotómica, en tal sentido, la investigación posee variable independiente principal y variables especificas en una escala de intervalo y variable dependiente dicotómica, que sitúa al estudio para comprobar la hipótesis con el modelo de regresión logística binaria Logit, en ese entender por considerarse un modelo de probabilidades no requiere pruebas de normalidad. 
Por tanto, la variable dependiente: detección de actos de corrupción se encuentra codificada, como sigue:

Tabla 3

Codificación de la variable dependiente

\begin{tabular}{cl}
\hline \multicolumn{2}{c}{ Codificación de detección de actos de corrupción } \\
\hline Valor original & Valor interno \\
No detecta & 0 \\
Sí detecta & 1 \\
\hline
\end{tabular}

Nota. Datos del cuestionario objeto del estudio

De la información recolectada se procede analizar los indicadores de la variable independiente en relación a la variable dependiente, en este caso, se analiza la influencia de la experiencia profesional del contador público colegiado en la detección (descubrimiento) de los actos de corrupción.

\section{Tabla 4}

Influencia de los años experiencia profesional del contador en la detección de actos de corrupción

\begin{tabular}{lccccccc}
\hline & \multicolumn{3}{c}{ Detección de la corrupción } & \multicolumn{2}{c}{ Total } \\
\cline { 2 - 6 } Años de experiencia (a partir de la colegiatura) & \multicolumn{2}{c}{ No detecta } & \multicolumn{2}{c}{ Sí detecta } & & \\
\cline { 2 - 6 } & $\mathrm{n}^{\circ}$ & $\%$ & $\mathrm{n}^{\circ}$ & $\%$ & $\mathrm{n}^{\circ}$ & $\%$ \\
\hline No tengo experiencia laboral & 3 & 60 & 2 & 40 & 5 & 100 \\
De 1 a 5 años & 59 & 44.36 & 74 & 55.64 & 133 & 100 \\
De 6 a 10 años & 19 & 29.23 & 46 & 70.77 & 65 & 100 \\
De 11 a más años & 8 & 20.51 & 31 & 79.49 & 39 & 100 \\
\hline Total & 89 & 36.78 & 153 & 63.22 & 242 & 100 \\
\hline
\end{tabular}

Nota. Datos extraídos del cuestionario dirigido al contador público colegiado de la región Moquegua (2020)

Conforme se detalla en la Tabla 4, los contadores públicos colegiados de la región Moquegua con experiencia profesional de once años a más detectan los actos de corrupción en $79.49 \%$, seguido de los contadores de seis a diez años de experiencia que detectan los actos de corrupción en $70.77 \%$, así como de los profesionales en Contabilidad de uno a cinco años de experiencia que detectan en $55.64 \%$ y finalmente los contadores que no poseen años de experiencia detectan los actos de corrupción en 40 $\%$ en relación de los contadores en la misma escala y rango de edad que no detectan los hechos ilícitos. Asimismo, es necesario destacar que, de acuerdo a la cantidad, en mayor proporcionalidad detectan los actos de corrupción los contadores que poseen de seis a diez años de experiencia.

Análisis de las ramas de especialización del contador y su influencia en la detección de la corrupción. 
Tabla 5

Influencia de ramas de especialización del contador en la detección de actos de corrupción

\begin{tabular}{|c|c|c|c|c|c|c|}
\hline \multirow{3}{*}{$\begin{array}{l}\text { Especialización del contador público } \\
\text { colegiado }\end{array}$} & \multicolumn{4}{|c|}{ Detección de actos de corrupción } & \multirow{2}{*}{\multicolumn{2}{|c|}{ Total }} \\
\hline & \multicolumn{2}{|c|}{ No detecta } & \multicolumn{2}{|c|}{ Sí detecta } & & \\
\hline & $\mathrm{n}^{\circ}$ & $\%$ & $\mathrm{n}^{\circ}$ & $\%$ & $\mathrm{n}^{\circ}$ & $\%$ \\
\hline $\begin{array}{l}\text { Gestión administrativa (tesorero, liquidador } \\
\text { financiero, logístico, proyectos de inversión) }\end{array}$ & 67 & 41.61 & 94 & 58.39 & 161 & 100 \\
\hline Auditor & 7 & 33.33 & 14 & 66.67 & 21 & 100 \\
\hline $\begin{array}{l}\text { Contador General (Finanzas, Tributaria, } \\
\text { NIIF, Costos y Presupuestos, Integrador } \\
\text { Contable) }\end{array}$ & 13 & 22.81 & 44 & 77.19 & 57 & 100 \\
\hline Perito & 2 & 66.67 & 1 & 3.33 & 3 & 100 \\
\hline Total & 89 & 36.78 & 153 & 63.22 & 242 & 100 \\
\hline
\end{tabular}

Nota. Datos extraídos del cuestionario dirigido al contador público colegiado de la región Moquegua (2020)

De acuerdo a la tabla anterior, los contadores públicos colegiados de la región Moquegua con especialización como Contador General en Finanzas, Tributaria, NIIF, Costos y Presupuestos e Integrador Contable, detectan actos de corrupción en $77.19 \%$, seguido de los contadores con especialización en Auditoría que detectan los actos de corrupción en 66.67 \% y los contadores especializados en Gestión Administrativa como son: administrador, tesorero, liquidador financiero, logístico y proyectos de inversión que detectan en $58.39 \%$.

Análisis de las horas de capacitación del contador y su relación con la detección de los hechos ilícitos.

Tabla 6

Influencia de la capacitación del contador en la detección de actos de corrupción

\begin{tabular}{lcccccccc}
\hline \multirow{2}{*}{$\begin{array}{l}\text { Horas de capacitación } \\
\text { (últimos 5 años) }\end{array}$} & \multicolumn{3}{c}{ Detección de actos de corrupción } & \multicolumn{2}{c}{ Total } \\
\cline { 2 - 6 } & \multicolumn{2}{c}{ No detecta } & \multicolumn{2}{c}{ Sí detecta } & & \\
\cline { 2 - 6 } & $\mathrm{n}^{\circ}$ & $\%$ & $\mathrm{n}^{\circ}$ & $\%$ & $\mathrm{n}^{\circ}$ & $\%$ \\
\hline No cuento con cursos de capacitación & 7 & 53.85 & 6 & 46.15 & 13 & 100 \\
Poco (1 a 100 horas de capacitación) & 29 & 38.67 & 46 & 61.33 & 75 & 100 \\
Regular (101 a 500 horas de capacitación) & 45 & 41.67 & 63 & 58.33 & 108 & 100 \\
Constante (501 a más horas de capacitación) & 8 & 17.39 & 38 & 82.61 & 46 & 100 \\
\hline Total & 89 & 36.78 & 153 & 63.22 & 242 & 100 \\
\hline
\end{tabular}

Nota. Datos extraídos del cuestionario dirigido al contador público colegiado de la región Moquegua (2020)

De acuerdo a la información procesada, los contadores públicos colegiados de la región Moquegua con capacitación constante de 501 a más horas en los últimos 5 años detectan los actos de corrupción en $82.61 \%$, sin embargo, en relación a cantidades, los contadores con regular capacitación detectan en mayor proporcionalidad los actos de corrupción. 
Análisis de la influencia de la edad del contador en la detección de los actos de corrupción.

Tabla 7

Influencia de la edad del contador en la detección de actos de corrupción

\begin{tabular}{lccccccc}
\hline & \multicolumn{3}{c}{ Detección de actos de corrupción } & \multicolumn{2}{c}{ Total } \\
\cline { 2 - 6 } Edad del contador público colegiado & \multicolumn{2}{c}{ No detecta } & \multicolumn{2}{c}{ Sí detecta } & & \\
\cline { 2 - 6 } & $\mathrm{n}^{\circ}$ & $\%$ & $\mathrm{n}^{\circ}$ & $\%$ & $\mathrm{n}^{\circ}$ & $\%$ \\
\hline De 23 a 30 años & 36 & 51.43 & 34 & 48.57 & 70 & 100 \\
De 31 a 45 años & 46 & 32.86 & 94 & 67.14 & 140 & 100 \\
De 46 a 60 años & 5 & 17.24 & 24 & 82.76 & 29 & 100 \\
De 61 a más años de edad & 2 & 66.67 & 1 & 33.33 & 3 & 100 \\
\hline Total & 89 & 36.78 & 153 & 63.22 & 242 & 100 \\
\hline
\end{tabular}

Nota. Datos extraídos del cuestionario dirigido al contador público colegiado de la región Moquegua (2020)

Según los resultados presentados en la Tabla 7, el contador público colegiado de la región Moquegua que se encuentra entre la edad de 46 a 60 años detecta los actos de corrupción en $82.76 \%$, seguido de los contadores de 31 a 45 años de edad que detectan en $67.14 \%$, no obstante, de acuerdo a la cantidad, los contadores que oscilan entre 31 a 45 años detectan en mayor proporcionalidad los actos de corrupción.

Análisis de la influencia del tipo de género del contador (femenino o masculino) en el descubrimiento de los actos de corrupción.

\section{Tabla 8}

Influencia del tipo de género del contador en la detección de actos de corrupción

\begin{tabular}{lcccccccc}
\hline \multirow{2}{*}{ Género del contador } & \multicolumn{3}{c}{ Detección de actos de corrupción } & \multicolumn{2}{c}{ Total } \\
\cline { 2 - 6 } & \multicolumn{2}{c}{ No detecta } & \multicolumn{2}{c}{ Sí detecta } & & \\
\cline { 2 - 6 } & $\mathrm{n}^{\circ}$ & $\%$ & $\mathrm{n}^{\circ}$ & $\%$ & $\mathrm{n}^{\circ}$ & $\%$ \\
\hline Masculino & 24 & 26.97 & 65 & 73.03 & 89 & 100 \\
Femenino & 65 & 42.48 & 88 & 57.52 & 153 & 100 \\
\hline Total & 89 & 36.78 & 153 & 63.22 & 242 & 100 \\
\hline
\end{tabular}

Nota. Datos extraídos del cuestionario dirigido al contador público colegiado de la región Moquegua (2020)

Los contadores públicos colegiados de la región Moquegua de género masculino detectan los actos de corrupción en $73.03 \%$, sin embargo, en cantidades los contadores de género femenino detectan los actos de corrupción en mayor proporcionalidad.

En forma complementaria, se analizaron: la posición, factores, instancias donde denuncia el contador y los resultados de las denuncias realizadas por el profesional. A continuación, se presenta el análisis de la posición del contador público colegiado en lo referente a la denuncia de los hechos de corrupción detectados. 


\section{Tabla 9}

Posición de los contadores relacionados a la denuncia de los actos de corrupción detectados

\begin{tabular}{lcc}
\hline $\begin{array}{l}\text { ¿Usted denuncia y/o comunica las acciones } \\
\text { ilícitas detectadas? }\end{array}$ & $\mathrm{n}^{\circ}$ & $\%$ \\
\hline Nunca & 40 & 26.32 \\
Algunas veces & 62 & 40.79 \\
Casi siempre & 12 & 7.89 \\
Siempre & 38 & 25 \\
\hline Total & 152 & 100 \\
\hline
\end{tabular}

Nota. Datos extraídos del cuestionario dirigido al contador público colegiado de la región Moquegua (2020)

De los actos de corrupción detectados por los contadores en el desarrollo de sus actividades profesionales el $40.79 \%$ presentó denuncia alguna vez, seguido del $25 \%$ que presentó denuncia siempre y el $26.32 \%$ que nunca presentó denuncia, se considera a este último como de mayor importancia.

Evaluación de los factores por los que el contador público colegiado no denuncia los actos de corrupción detectados.

Tabla 10

Razones por las que los contadores no denuncian los actos de corrupción

\begin{tabular}{lcc}
\hline ¿Por qué no comunicó y/o denunció la acción ilícita detectada? & $\mathrm{n}^{\circ}$ & $\%$ \\
\hline Pérdida de tiempo no se adoptan medidas y prefiero evitar problemas & 38 & 36.19 \\
No me corresponde denunciar & 15 & 14.29 \\
Por conservar mí trabajo & 18 & 17.14 \\
No se contó con evidencia (falta de pruebas) & 34 & 32.38 \\
\hline Total & 105 & 100 \\
\hline
\end{tabular}

Nota. Datos extraídos del cuestionario dirigido al contador público colegiado de la región Moquegua (2020)

Según los datos procesados del porcentaje de contadores que no denuncian y/o comunican los actos de corrupción detectados, el $36.19 \%$ de los contadores considera que no denuncia porque es pérdida de tiempo denunciar, no se adoptan medidas y prefieren evitar problemas, seguido de aquellos que no denuncian porque no cuentan con evidencia suficiente (falta de pruebas), el $17.14 \%$ refiere que no denuncia por conservar su trabajo y finalmente el $14.29 \%$ indica que no les corresponde denunciar.

Análisis de las instancias donde denuncia el contador público colegiado los actos de corrupción detectados. 


\section{Tabla 11}

Instancias donde denuncian los contadores los actos de corrupción detectados

\begin{tabular}{lcc}
\hline $\begin{array}{l}\text { ¿A qué instancia comunicó y/o presentó la denuncia de las acciones ilícitas } \\
\text { detectadas? }\end{array}$ & $\mathrm{n}^{\circ}$ & $\%$ \\
\hline Jefe inmediato y/o titular de la entidad & 100 & 89.29 \\
Medios de comunicación & 2 & 1.79 \\
Contraloría & 5 & 4.46 \\
Poder Judicial & 5 & 4.46 \\
\hline Total & 112 & 100 \\
\hline
\end{tabular}

Nota. Datos extraídos del cuestionario dirigido al contador público colegiado de la región Moquegua (2020)

Los actos de corrupción detectados por los contadores son denunciados y/o comunicados en $89.29 \%$ al jefe inmediato y/o al titular de la entidad, el $4.46 \%$ son denunciados en la Contraloría General de la República y el Poder Judicial respectivamente y finalmente el $1.79 \%$ son denunciados ante los medios de comunicación.

Evaluación de los resultados de los actos de corrupción denunciados por el contador público colegiado.

Tabla 12

Resultado de los actos de corrupción denunciados por los contadores

\begin{tabular}{llc}
\hline ¿Cuál fue el resultado de su denuncia, se determinaron responsabilidades? & $\mathrm{n}^{\circ}$ & $\%$ \\
\hline Muy efectivo se determinó responsabilidad y sanciones ejemplares & 10 & 8.93 \\
Efectivo se determinó responsabilidad administrativa & 26 & 23.21 \\
Denuncia fue archivado & 10 & 8.93 \\
Nada efectivo no se investigó & 66 & 58.93 \\
\hline Total & 112 & 100 \\
\hline
\end{tabular}

Nota. Datos extraídos del cuestionario dirigido al contador público colegiado de la región Moquegua (2020)

De las denuncias realizadas por el contador público colegiado por los actos de corrupción detectados, el $58.93 \%$ refiere que no fue nada efectivo (no se investigó), el $8.93 \%$ indica que la denuncia fue archivada, sin embargo, el $23.21 \%$ señala que la denuncia fue efectiva, se determinó responsabilidad administrativa y el $8.93 \%$ indica que la denuncia fue muy efectiva se determinaron responsabilidades y sanciones ejemplares.

\section{Prueba de hipótesis}

Para la comprobación de los resultados, se utilizó el estadístico no paramétrico que busca la implicancia o influencia entre los perfiles profesionales y su relación con la variable dependiente: detección de la corrupción, asimismo, para el contraste de hipótesis se empleó la prueba de regresión logística binaria, ya que los datos para el modelamiento son de carácter cualitativo nominal, orientado al modelo de 
regresión logística binario, que se procesó en el reporte del software estadístico SPSS ver. 25.

Para la aplicación de un modelo de regresión, es necesario corroborar que el modelo se ajusta efectivamente a los datos objeto de análisis.

La prueba Omnibus, valora si la introducción de las variables independientes logra un incremento significativo del ajuste global de los datos.

\section{Tabla 13}

Prueba Omnibus

\begin{tabular}{lcccc}
\hline \multicolumn{5}{c}{ Pruebas Omnibus de coeficientes de modelo } \\
\hline \multirow{4}{*}{ Paso 1 } & Chi-cuadrado & Gl & Sig. \\
& Paso & 34.274 & 12 & .001 \\
& Bloque & 34.274 & 12 & .001 \\
& Modelo & 34.274 & 12 & .001 \\
\hline
\end{tabular}

Nota. Procesado en software estadístico SPSS ver. 25

Ho: El modelo no se ajusta a los datos

H1: El modelo se ajusta a los datos

El modelo de regresión logística se ajusta al conjunto de datos analizados dado que el valor-p es menor que el 0.05 .

Contraste de la primera hipótesis específica planteada que detalla que la experiencia profesional del contador público colegiado influye significativamente en la detección de los actos de corrupción en la región Moquegua, 2019.

\section{Tabla 14}

Variables en la ecuación de la variable independiente experiencia profesional del contador en relación a la detección de los hechos de corrupción

\begin{tabular}{lcccccc}
\hline \multicolumn{7}{c}{ Variables en la ecuación } \\
\hline $\begin{array}{l}\text { Años de experiencia (a partır de la } \\
\text { colegiatura) }\end{array}$ & B & $\begin{array}{c}\text { Error } \\
\text { estándar }\end{array}$ & Wald & G1 & Sig. & Exp(B) \\
\hline $\begin{array}{l}\text { No cuenta con experiencia } \\
\text { profesional }\end{array}$ & & & 10.062 & 3 & .018 & \\
{$[1$ a 5> } & -1.760 & .995 & 3.127 & 1 & .077 & .172 \\
[6 a 10> & -1.128 & .433 & 6.778 & 1 & .009 & .324 \\
De 11 a más años & -.470 & .481 & .955 & 1 & .328 & .625 \\
Constante & 1.355 & .397 & 11.667 & 1 & .001 & 3.875 \\
\hline
\end{tabular}

Nota. a. Variables especificadas en el paso 1: X1 Años de Experiencia (a partir de la colegiatura)

El valor-p de 0,009 menor a 0.05 de nivel de significancia obtenido en la tabla precedente, expone que la experiencia profesional de seis a diez años que posee el contador público colegiado influye significativamente en la detección de los actos de corrupción en la región Moquegua.

Contraste de la segunda hipótesis específica que plantea que la especialización del contador público 
colegiado influye significativamente en la detección de los actos de corrupción en la región Moquegua, 2019.

\section{Tabla 15}

Variables en la ecuación de la variable independiente especialización del contador público colegiado en relación a la detección de los hechos ilícitos

\begin{tabular}{|c|c|c|c|c|c|c|}
\hline \multicolumn{7}{|c|}{ Variables en la ecuación } \\
\hline $\begin{array}{l}\text { Especialización del contador } \\
\text { público } \\
\text { Paso } 1^{\text {a }}\end{array}$ & B & $\begin{array}{c}\text { Error } \\
\text { estándar }\end{array}$ & Wald & Gl & Sig. & $\operatorname{Exp}(B)$ \\
\hline $\begin{array}{l}\text { Gestión administrativa (tesorero, } \\
\text { liquidador financiero, logístico, } \\
\text { proyectos de inversión) }\end{array}$ & & & 6.648 & 2 & .036 & \\
\hline Auditor & .373 & .489 & .583 & 1 & .445 & 1.453 \\
\hline $\begin{array}{l}\text { Contador General (Finanzas, } \\
\text { Tributaria, NIIF, Costos y } \\
\text { Presupuestos, Integrador Contable) }\end{array}$ & .899 & .353 & 6.490 & 1 & .011 & 2.458 \\
\hline Constante & .320 & .158 & 4.087 & 1 & .043 & 1.377 \\
\hline
\end{tabular}

Nota. a. Variables especificadas en el paso 1: X2 Especialización del contador público colegiado.

La especialización del contador público colegiado como Contador General, especialista en Finanzas, Tributaria, Normas Internacionales de Información Financiera, Costos, Presupuestos e Integrador Contable, logra un valor-p de 0.011 menor a 0,05 , en tal sentido, el perfil profesional de especialización influye significativamente en el descubrimiento y/o detección de los actos de corrupción en la región Moquegua.

Contraste de la tercera hipótesis específica que afirma que la capacitación del contador público colegiado influye significativamente en la detección de los actos de corrupción en la región Moquegua, 2019.

\section{Tabla 16}

Variables en la ecuación de la variable independiente capacitación del contador público colegiado en relación a la detección de la corrupción

\begin{tabular}{lcccccc}
\hline \multicolumn{7}{c}{ Variables en la ecuación } \\
\hline $\begin{array}{l}\text { Horas de capacitación - últimos 5 } \\
\text { años } \\
\text { Paso 1 }\end{array}$ & B & $\begin{array}{c}\text { Error } \\
\text { estándar }\end{array}$ & Wald & Gl & Sig. & Exp(B) \\
\hline No cuento con capacitación & & & 9.510 & 3 & .023 & \\
Poco [1 a 100> & .615 & .605 & 1.036 & 1 & .309 & 1.851 \\
Regular [101 a 500> & .491 & .590 & .692 & 1 & .405 & 1.633 \\
$\begin{array}{l}\text { Capacitación constante < a 501 } \\
\text { horas }\end{array}$ & 1.712 & .679 & 6.362 & 1 & .012 & 5.542 \\
Constante & -1.54 & .556 & .077 & 1 & .782 & .857 \\
\hline
\end{tabular}

Nota. Paso 1: X3 Horas de capacitación - últimos 5 años del contador público colegiado 
La capacitación del contador público colegiado que cuenta con una capacitación constante de 501 a más horas en los últimos cinco años, influye significativamente en el descubrimiento de los hechos ilícitos (corrupción) en la región Moquegua, toda vez que el valor-p alcanzado es de 0.012 menor a 0.05 .

Contraste de la cuarta hipótesis específica planteada que establece que la edad del contador público colegiado influye significativamente en la detección de los actos de corrupción en la región Moquegua, 2019.

\section{Tabla 17}

Variables en la ecuación de la variable independiente edad del contador público colegiado en relación a la detección de los hechos de corrupción

\begin{tabular}{lcccccc}
\hline \multicolumn{7}{c}{ Variables en la ecuación } \\
\hline $\begin{array}{l}\text { Edad del contador público } \\
\text { Paso 1 }\end{array}$ & B & $\begin{array}{c}\text { Error } \\
\text { estándar }\end{array}$ & Wald & Gl & Sig. & Exp(B) \\
\hline De 61 a más años & & & 12.520 & 3 & .006 & \\
{$[23$ a 30> } & -2.262 & 1.320 & 2.937 & 1 & .087 & .104 \\
{$[31$ a 45> } & -1.626 & .547 & 8.844 & 1 & .003 & .197 \\
{$[46$ a 60> } & -.854 & .523 & 2.661 & 1 & .103 & .426 \\
Constante & 1.569 & .492 & 10.182 & 1 & .001 & 4.800 \\
\hline
\end{tabular}

Nota. a. Variables especificadas en el paso 1: X4 Edad del contador público colegiado

La edad comprendida entre 31 a 45 años del profesional en Contabilidad colegiado influye significativamente en el descubrimiento de los actos de corrupción en la región Moquegua, dado que el valor-p calculado es de 0.003 menor a 0.05 de nivel de significancia.

Contraste de la quinta hipótesis específica que plantea que el género del contador público colegiado influye significativamente en la detección de los actos de corrupción en la región Moquegua, 2019.

Tabla 18

Variables en la ecuación de la variable independiente género del contador público colegiado en relación a la variable dependiente

\begin{tabular}{lcccccc}
\hline \multicolumn{7}{c}{ Variables en la ecuación } \\
\hline $\begin{array}{l}\text { Género del contador } \\
\text { Paso 1 }\end{array}$ & $\mathrm{B}$ & $\begin{array}{c}\text { Error } \\
\text { estándar }\end{array}$ & Wald & Gl & Sig. & Exp(B) \\
\hline Masculino & .693 & .289 & 5.737 & 1 & .017 & 2.000 \\
Constante & .303 & .164 & 3.431 & 1 & .064 & 1.354 \\
\hline
\end{tabular}

Nota. a. Variables especificadas en el paso 1: X5 Género del contador

El contador público colegiado de género masculino influye significativamente en el descubrimiento de los hechos ilegítimos relacionados con la corrupción en la región Moquegua, 2019, toda vez que alcanza valor-p de 0.017 menor a 0.05 de nivel de significancia. 
Contraste de la hipótesis general planteada que detalla que el perfil profesional del contador público colegiado influye significativamente en la detección de los actos de corrupción en la región Moquegua, 2019.

\section{Tabla 19}

Variables en la ecuación de la variable independiente perfil del contador público colegiado en relación a la variable dependiente detección de los actos de corrupción

\begin{tabular}{|c|c|c|c|c|c|c|}
\hline \multicolumn{7}{|c|}{ Variables en la ecuación } \\
\hline $\begin{array}{l}\text { Perfil profesional del contador } \\
\text { público } \\
\text { Paso } 3^{\text {a }} \\
\end{array}$ & B & $\begin{array}{l}\text { Error } \\
\text { estándar }\end{array}$ & Wald & Gl & Sig. & $\operatorname{Exp}(B)$ \\
\hline \multicolumn{7}{|c|}{ Especialización del contador público colegiado } \\
\hline Gestión administrativa & & & 5.317 & 2 & .070 & \\
\hline Auditor & -.825 & .368 & 5.031 & 1 & .025 & .438 \\
\hline $\begin{array}{l}\text { Contador General (Finanzas, } \\
\text { Tributaria, NIIF, Costos y } \\
\text { Presupuestos, Integrador Cont }\end{array}$ & -.366 & .602 & .370 & 1 & .043 & .693 \\
\hline \multicolumn{7}{|c|}{ Horas de capacitación - últimos 5 años } \\
\hline $\begin{array}{l}\text { No cuento con cursos de } \\
\text { capacitación }\end{array}$ & & & 9.640 & 3 & 0.22 & \\
\hline Poco $[1$ a $100>$ & -1.480 & .706 & 4.394 & 1 & 0.36 & .228 \\
\hline Regular [101 a 500> & -1.024 & .477 & 4.620 & 1 & 0.32 & .359 \\
\hline Constante $<$ a 501 horas & -1.368 & .454 & 9.090 & 1 & 0.03 & .255 \\
\hline \multicolumn{7}{|c|}{ Edad del contador público colegiado } \\
\hline De 61 a más años & & & 11.135 & 3 & .011 & \\
\hline$[23$ a $30>$ & -2.561 & 1.368 & 3.504 & 1 & .061 & .077 \\
\hline$[31$ a 45> & -1.659 & .567 & 8.556 & 1 & .003 & .190 \\
\hline$[46$ a $60>$ & -.964 & .538 & 3.214 & 1 & .073 & .381 \\
\hline Constante & 3.306 & .719 & 21.130 & 1 & .000 & 27.278 \\
\hline
\end{tabular}

Nota. a. Variables especificadas en el paso 1: X1 Años de experiencia, X2 Especialización del contador público colegiado, X3 Horas de capacitación - últimos 5 años, X4 Edad del contador público colegiado, X5 Género del contador. Método de entrada de Wald.

El perfil profesional del contador público colegiado influye significativamente en la detección de los actos de corrupción en la región Moquegua, 2019, toda vez que el perfil profesional de contador público con especialidad de Contador General, especialista en Finanzas, Tributaria, Normas Internacionales de Información Financiera, Costos, Presupuestos e Integrador Contable, obtiene valor-p de 0.043, el perfil profesional del contador público colegiado con capacitación constante de 501 a más horas, logra valor-p de 0.003 y finalmente el perfil profesional del contador público colegiado con rango de edad de 31 a 45 años obtiene valor-p de 0.003 , en general menores a 0.05 de nivel de significancia. 


\section{DISCUSIÓN}

Según los resultados del contraste de la hipótesis general, el perfil profesional del contador público colegiado influye significativamente en la detección de los hechos de corrupción en la región Moquegua, cuando el profesional en Contabilidad cuenta con especialización de Contador General, especialista en Finanzas, Tributaria, Normas Internacionales de Información Financiera, Costos, Presupuestos e Integrador Contable, posee capacitación constante de 501 a más horas en los últimos cinco años y que se encuentra en el rango de edad de 31 a 45 años, toda vez que, en las categorizaciones detalladas por perfil profesional del contador público colegiado el valor-p es menor a 0.05 de nivel de significancia, el cual difiere de los resultados del contraste de las cinco hipótesis específicas en forma independiente, en vista de que, además de los tres perfiles identificados incluye, para la detección de actos de corrupción, la experiencia profesional de seis a diez años del contador público colegiado y el contador de género masculino con más probabilidad de detectar los hechos ilícitos, por cuanto que en el contraste de datos efectuado se obtiene como resultado que el valor-p es menor a 0.05 de nivel de significancia en ambos casos.

Asimismo, es necesario resaltar que el análisis de datos presentado mediante los cuadros de Excel y gráficos amplía más el perfil profesional del contador público colegiado que influye en el descubrimiento de los actos corrupción, se reafirma que el contador que posee de seis a diez años de experiencia detecta los actos de corrupción en $70.77 \%$. Sin embargo, expone también que el profesional en Contabilidad, de once años a más de experiencia profesional, detecta los actos de corrupción en $79.49 \%$. En relación al perfil profesional de especialización que influye en la detección de actos de corrupción, el contador público colegiado con especialización de Contador General en Finanzas, Tributaria, NIIF, Costos y Presupuestos e Integrador Contable, detecta los actos de corrupción en $77.19 \%$ y además revela que los contadores con especialización de Auditoría detectan los actos de corrupción en 66.67 \% y sin excluir aquellos contadores especializados en Gestión Administrativa como: administrador, tesorero, logístico, liquidador financiero y proyectos de inversión, que detectan los actos de corrupción en 58.39 $\%$. En lo referente al perfil profesional de capacitación, efectivamente, los contadores con capacitación constante de 501 a más horas, en los últimos 5 años, detectan los actos de corrupción en $82.61 \%$. En lo relacionado a la edad del profesional en Contabilidad, se amplía el rango de edad, ya que el contador de 31 a 45 años de edad detecta los actos de corrupción en $67.14 \%$ y los contadores de 46 a 60 años edad detectan los actos de corrupción en un $82.76 \%$. Finalmente, en el perfil profesional de género del contador público colegiado se reafirma que efectivamente el contador de género masculino detecta los actos de corrupción en $73.03 \%$.

Los párrafos precedentes dan cuenta que el contraste de hipótesis general no considera el perfil profesional del contador público colegiado de experiencia profesional y género como influencia significativa para la detección de los hechos de corrupción. Sin embargo, de acuerdo al contraste de la primera hipótesis específica, la experiencia profesional del contador público colegiado de seis a diez años sí influye significativamente en la detección de actos de corrupción. Esto es ratificado en el análisis de resultados presentados en los cuadros Excel y sustentado teóricamente por Cart \& Toutin (1998), quienes indican que la experiencia profesional permite la multiplicidad de la actividad profesional, en el proceso de construcción de las capacidades que juega un papel esencial. Esta afirmación se ajusta a las actividades diarias de los profesionales y en el caso específico a la población objeto de análisis, que son profesionales habilitados en ejercicio, que prestan sus servicios profesionales en los diferentes campos laborales, hechos que les permiten adquirir competencias y habilidades profesionales en forma permanente. En tal sentido, es preciso afirmar que la experiencia profesional sí influye en la detección de actos de corrupción, y en relación al género del contador de acuerdo al análisis de datos realizado con cuadros Excel, los contadores de género masculino sí influyen en el hallazgo de los actos de corrupción en la región Moquegua.

Del mismo modo, es necesario detallar que la especialización, según Zapata \& Sigala (2010), brinda al profesional cantidad, profundidad y variedad de habilidades y conocimientos para responder 
adecuadamente a la diversidad de problemas, aseveración que respalda el resultado de que la especialización de Contador General, especialista en Finanzas, Tributaria, Normas Internacionales de Información Financiera, Costos, Presupuestos, Integrador Contable y Auditor influye en la detección de actos de corrupción. De igual forma, con respecto a la capacitación, la directiva de capacitación de la Escuela Nacional de Control (2019) detalla que el proceso de capacitación permite ampliar y mejorar las habilidades, conocimientos y actitudes, pauta que refrenda los resultados que establecen que la capacitación constante de 501 a más horas en los últimos cinco años influye en el hallazgo de los actos de corrupción; y referente a la edad, el contador de 31 a 45 años que influye a la detección de hechos ilícitos, Fernández (2002) señala que la edad comprendida de 30 a 45 años se caracteriza por el ascenso, crecimiento, seguridad y desarrollo, donde el profesional es contribuidor independiente, definición que respalda los resultados obtenidos en relación al rango de edad.

\section{CONCLUSIONES}

Se concluye que el perfil profesional del contador público colegiado que influye significativamente en la detección de los actos de corrupción en la región Moquegua es el contador público colegiado que posee experiencia profesional de seis a diez años, que ostenta la especialización de Contador General, especialista en Finanzas, Tributaria, Normas Internacionales de Información Financiera, Costos, Presupuestos, Integrador Contable y Auditor, con capacitación constante de 501 a más horas en los últimos cinco años y que se encuentra entre la edad de 31 a 45 años, toda vez que, en el contraste de hipótesis general, obtuvieron valor-p menor al nivel de significancia de 0.05 , conforme se detalla a continuación: el perfil profesional de especialización del contador público colegiado con especialidad de Contador General, especialista en Finanzas, Tributaria, Normas Internacionales de Información Financiera, Costos, Presupuestos e Integrador Contable, logra valor-p de 0.043, de igual forma el perfil profesional de capacitación del contador público colegiado con capacitación constante de 501 a más horas, obtiene valor-p de 0,003 y el perfil profesional de rango de edad del contador público colegiado de 31 a 45 años logra valor-p de 0,003. De igual forma, en lo relacionado al perfil profesional del contador, en cuanto a su experiencia profesional, se encuentra sustentado con el contraste de la primera hipótesis específica, donde la experiencia profesional del contador público colegiado de seis a diez años obtiene valor-p de 0.009 menor a 0.05 de nivel de significancia, el cual se encuentra ratificado por el análisis de resultados presentados en los cuadros Excel, donde el contador público colegiado de seis a diez años de experiencia profesional detecta los actos de corrupción en $70.77 \%$. Finalmente, cuenta con sustento teórico de Cart \& Toutin (1998) quienes refieren que, en el proceso de construcción de las capacidades, la experiencia profesional juega un papel esencial, toda vez que permite la multiplicidad de la actividad profesional, razón por la que se considera la experiencia profesional como parte del perfil profesional del contador para la detección de los actos de corrupción. Del mismo modo, el perfil profesional de especialización, capacitación y edad del contador que influye significativamente en la detección de actos de corrupción, según el contraste de hipótesis general, se encuentran sustentados teóricamente por Zapata \& Sigala (2010), quienes señalan que la especialización brinda al profesional profundidad, cantidad y variedad de habilidades y conocimientos para responder adecuadamente a la diversidad de problemas. En lo relacionado al perfil de capacitación, es necesario considerar teóricamente que el proceso de capacitación permite ampliar y mejorar las habilidades, conocimientos y actitudes (Directiva de capacitación de la Escuela Nacional de Control, 2019). Finalmente, el perfil de edad del contador público colegiado se encuentra amparado en lo referido por Fernández (2002), quien señala que el profesional es contribuidor e independiente y se caracteriza por el ascenso, crecimiento, seguridad y desarrollo en la edad comprendida de 30 a 45 años. 


\section{Figura 1}

Perfil profesional de contador público colegiado que influye significativamente en la detección y/o hallazgo de los actos de corrupción en la región Moquegua

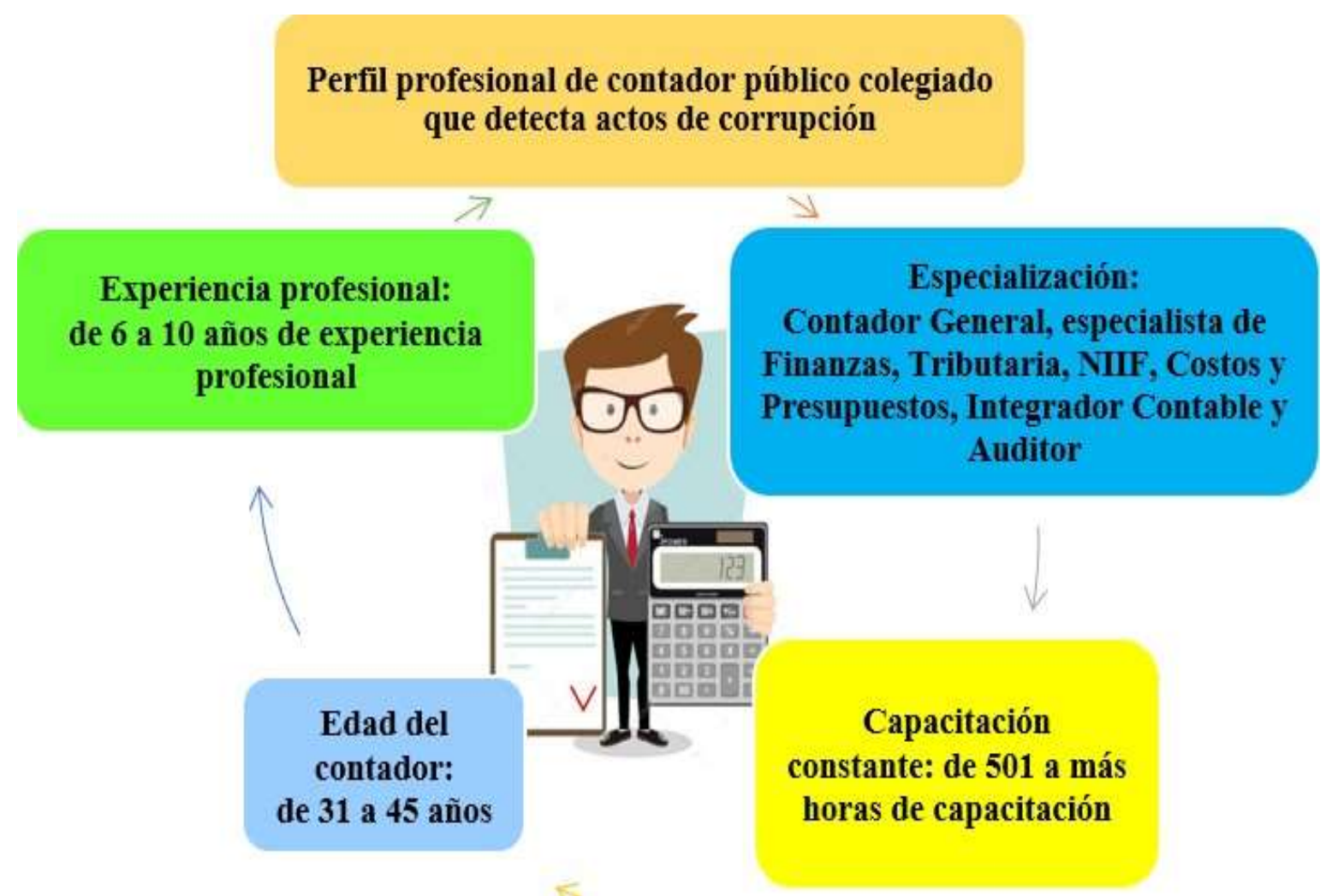

Nota. La figura presenta el perfil profesional de contador público colegiado que influye significativamente en el descubrimiento de los actos de corrupción. Fuente: Cuestionario dirigido al contador público colegiado de la región Moquegua (2020)

En relación al perfil de género del contador público colegiado, de acuerdo al resultado de las probabilidades del modelo de regresión logística binaria Logit, análisis de datos y fundamentación teórica, el perfil de género del contador no influye en el descubrimiento de los actos de corrupción, resultado que se ajusta a la realidad de la región Moquegua, toda vez que la participación del profesional de género femenino y masculino es equitativo y no existe diferencia significativa en su desenvolvimiento profesional, aseveración que se encuentra fundamentado teóricamente en lo señalado por Aldana (2013), quien indica que entre hombres y mujeres no hay diferencia considerable con respecto al grado de compromiso organizacional.

Una vez determinado el perfil profesional que influye en el hallazgo y/o detección de los actos de corrupción en la región Moquegua, es necesario comentar sobre los actos de corrupción detectados por los contadores que no son difundidos y reconocidos notablemente por la sociedad, que, de acuerdo a los resultados obtenidos, el $26.32 \%$ de los contadores que detectan hechos ilícitos nunca han presentado denuncia, el $40.79 \%$ presentó denuncia alguna vez y solo el $25 \%$ presenta denuncia de los hechos ilícitos detectados en el ejercicio de sus labores diarias. Este contexto exige el análisis de los actos de corrupción detectados por los contadores no denunciados, de los cuales el $36.19 \%$ no denuncia porque considera que es pérdida de tiempo, que no se adoptan medidas y prefieren evitar problemas, el $17.14 \%$ refiere que no denuncia por conservar su trabajo y el $14.29 \%$ señala que no le corresponde denunciar, en lo referente a los actos de corrupción denunciados y/o comunicados por los contadores, el 89.29 $\%$ denuncia y/o comunica el hecho ilícito al jefe inmediato y/o al titular de la entidad, solo el $4.46 \%$ denuncia al Poder Judicial y la Contraloría General de la República los actos de corrupción detectados 
y por último el $1.79 \%$ denuncia en los medios de comunicación. No obstante, de las comunicaciones y/o denuncias realizadas por el contador público colegiado, el $58.93 \%$ de los contadores refiere que no fue nada efectivo (no se investigó) y el $8.93 \%$ señala que la denuncia fue archivada, solo el 23.21 $\%$ de los contadores indica que la denuncia fue efectiva (se determinó responsabilidad administrativa) y el $8.93 \%$ de contadores indica que la denuncia fue muy efectiva, se determinaron responsabilidades y sanciones ejemplares. Situaciones que explican el por qué no trascienden los actos de corrupción detectados y denunciados por los contadores, en tal sentido, como parte del presente estudio se plantea propuesta de formación del perfil profesional del contador público colegiado con responsabilidad social en las diferentes etapas profesionales del contador, donde puedan recibir apoyo, asesoramiento y reconocimiento por hacer frente a las actividades ilícitas (corrupción) y ejercer con responsabilidad su profesión en beneficio de la entidad donde labora y la sociedad en general.

La propuesta tiene como finalidad establecer la formación del perfil profesional del contador público colegiado con responsabilidad social, al considerar sus competencias y su desenvolvimiento en las diferentes áreas de las entidades del ámbito público y privado, donde el profesional en Contabilidad independientemente de su labor profesional pueda fortalecer su responsabilidad para con la sociedad. En tal sentido, el perfil profesional del contador público colegiado con responsabilidad social, además de la experiencia profesional, capacitación, especialización y edad del contador, debe contar con capacitaciones anuales de ética profesional y responsabilidad social acreditado por el Colegio de Contadores Públicos, medidas estrictas que se deben adoptar para formar parte del Colegio Profesional, ya que en la actualidad la única condición para formar parte de la institución y adquirir una habilitación para el ejercicio de la profesión es el pago de derechos. No se considera la formación y/o constitución del perfil profesional del contador público colegiado con responsabilidad social como un aporte responsable ante la sociedad y que podría constituir instrumento esencial en la lucha contra la corrupción. De igual forma, la propuesta plantea cinco componentes, formación de perfil profesional, seguimiento de las actividades laborales del contador público colegiado, asesoramiento y apoyo a los contadores que denuncian hechos ilícitos en perjuicio de la sociedad, reconocimiento de los contadores que se desempeñan con responsabilidad social y sanción de los contadores que forman parte de los hechos ilícitos.

La formación de perfil profesional plantea que el profesional, antes de formar parte del Colegio Profesional, sea evaluado psicológicamente en relación a su actuar responsable para con la sociedad y de acuerdo a los resultados, se debe determinar su ingreso o su concientización a través de talleres semestrales durante los primeros años agremiación. Asimismo, para los miembros del Colegio Profesional en forma obligatorio se deben llevar a cabo los talleres psicológicos, ética profesional del contador y responsabilidad social en forma anual, además, debe ser evaluado al término de cada taller y debe constituir como requisito para habilitación profesional, propuesta que, de concretarse podría hacerse extensivo en todos los colegios profesionales a nivel nacional y permitiría en forma obligatoria a sus agremiados concientizarse sobre la ética profesional y responsabilidad social. En consecuencia, se podría constituir como instrumento clave para contrarrestar la corrupción, toda vez que todos los profesionales para el ejercicio de su profesión necesitan de una habilitación que en este caso estaría condicionado con la participación en talleres de concientización independientemente del pago de los derechos.

Asimismo, es necesario mencionar que muchos profesionales que detectan hechos ilícitos en el ejercicio de sus funciones no denuncian porque se encuentran condicionados con el puesto de trabajo y no cuentan con el apoyo de alguna organización en caso de denunciar y al contrario se ven perjudicados económicamente al perder su puesto laboral y enfrentar el proceso judicial, situaciones que han contribuido a la impunidad y el crecimiento diario en grandes escalas de la corrupción. En tal sentido, la propuesta presentada es trascendental, toda vez que convierte al Colegio Profesional en una organización de apoyo a los profesionales que deciden hacer frente a la corrupción además de concientizar para su prevención y convertirse así en una organización activa en la sociedad que debe ser considerado por el Gobierno para contrarrestar la corrupción. 


\section{REFERENCIAS}

Aldana, P. J. (2013). Compromiso Laboral de los Trabajadores del Área Central deuna Institución Bancaria que opera en la Ciudad de Guatemala, según Sexo [Tesis de Maestria, Universidad Rafael Landívar, Guatemala]. Repositorio institucional. http://biblio3.url.edu.gt/Tesario/2013/05/43/ Aldana-Jennifer.pdf

Alegría, C. O. (2016). El rol del Contador Público frente a la implementación de la responsabilidad social empresarial a nivel del sector empresarial en el Perú, período 2015-2016 [Tesis Doctoral, Universidad San Martín de Porres, Lima]. Repositorio institucional. https://hdl. handle.net/20.500.12727/2474.

Basílico, R. A. (2014). Negociaciones y Actividades Prohibidas a los Funcionarios Públicos y de los Abusos en el Ejercicio de su Función [Tesis Doctoral, Universidad de Sevilla, España]. Depósito de investigación. http://hdl.handle.net/11441/58681

Cart, B., \& Toutin, M. (1998). La production d'adaptabilité: Les modalités de construction de l'expérience professionnelle. In Stankiewicz F. (Ed.), 137-157.

Directiva de transferencias financieras para el desarrollo de programas formativos y de capacitación por medio de la Escuela Nacional de Control de 2019. (2019, 17 de enero). Contraloría General de la República. Resolución de Contraloría n. ${ }^{\circ}$ 021-2019-CG. https://www.contraloria.gob.pe/wps/ $\mathrm{wcm} /$ connect/fc402a82-596d-4937-9135-a02b18277033/RC_021-2019-CG-DIRECTIVA-0012019-CG.pdf? MOD=AJPERES\&CACHEID=fc402a82-596d-4937-9135-a02b18277033 .

Fernández, L. N. (2002). El desarrollo profesional de los trabajadores como ventaja competitiva de las empresas. Cuadernos de Gestión. 2( 1), 65 - 90.

Guillen, Z. A. (2017). Sistemas de Control y Prevención en las empresas sobre delitos de corrupción. [Tesis Doctoral, Universidad Rey Juan Carlos, Madrid - España]. Repositorio institucional de la Universidad / Dialnet. https://www.educacion.gob.es/teseo/imprimirFicheroTesis. do?idFichero=SseTgYcMw9U\%3D.

Hernandez, D. A. (2011). El perfil profesional del presidente municipal en el estado de México. Editores S.A.

Hérnandez, Sampieri Roberto; Fernández, Collado Carlos; Baptista, Lucio María del Pilar. (2014). Metodología de la investigación. Mc GRAW-HILL/Interamericana Editores S.A. de C.V. Sexta edición.

Lamas, M. (2000). Diferencias de sexo, género y diferencia sexual. Cuicuilco, 7(18), 1-24.

Luis, O. R. (2012). La contribución del Contador Público en la erradicación de la corrupción. Apuntes contables, $7-24$.

Maturana, R. (1997). Formación Humana y Capacitación. Dolmen Ediciones S.A. 5(2), 10 - 20.

Santos, G. E. (2018). El contador público como causa primera y última de la confianza pública. Contabilidad y Negocios. Escuela Profesional de Contabilidad - Universidad Peruana Unión. 13 (25), 109-124. Recuperado de https://doi.org/10.18800/contabilidad.201801.008.

Valpuesta, F. M. (2003). El Derecho civil. Codificación y Constitución. Tirant lo blanch: 4ta. edición.

Zapata, R.G.; Sigala, P. L. (2010). Tipología de la especialización del trabajo en la organización: Fundamentos para su propuesta. Visión Gerencial, (2), 430-442. Recuperado de https://www. redalyc.org/pdf/4655/465545889009.pdf. 